\title{
Research on Feature Extraction and Match Method based on the Surf Algorithm for Mobile Augmented Reality System
}

\author{
Fei Guo ${ }^{1, a}$, Xiao Luo ${ }^{1, b}$, Yi Liü, c \\ ${ }^{1}$ School of Information Engineering, Beijing Institute of Fashion Technology, Beijing 100029, China \\ aemail: lunwen95@163.com, bemail: Ixiaorow@sohu.com, 'email: gxyly@bift.edu.cn
}

Keywords: Surf, Augmented Reality, Image Matching, Feature Point, Mobile Device

\begin{abstract}
According to the characteristics of constrained mobile devices, an improved Surf feature extraction and matching algorithm is proposed. First, dynamic pyramid is established in order to meet the real-time requirements; then reducing redundant feature points and strengthening the feature points of big difference individuality is done based on the Niche ideas; finally improved classification BBF matching algorithm is used to speed the matching. The contrast experiment on Surf and its improved algorithm is done based on the iOS platform. The experimental results demonstrate that the improved Surf algorithm has strong robustness, fast speed, high accuracy, and lays the foundation for mobile augmented reality technology in video, animation, text, 3D virtual scene registration, so it has great application value.
\end{abstract}

\section{Introduction}

Augmented reality (AR) is a hot research direction of computer vision field in recent years, which has been applied in many fields such as medical, military, education, entertainment [1]. Augmented reality based on mobile terminal has been the future development direction with the development of mo-bile Internet technology and the popularity of smart mobile devices [2]. However, memory and computing speed of mobile devices more less than computer, therefore, to make the augmented reality technology better on mobile devices, it is must be first studied on the key of augmented reality according to the characteristics of mobile devices: features extraction and matching algorithm.

Feature extraction, matching, recognition technology is the focus of AR research. The existing feature registration techniques is mainly include based on special marker (Marker) and based on natural features(Markerless)[3]. Marker registration method is rapid and stable, but the maker need to been shown completely in a video, and it affects the visual effects because of the markers are generally black and white image, so marker registration method has great limitation. The commonly recognition method based on natural features consist of Sift(Scale Invariant Feature Transform), Surf(Speeded-up Robust Feature), Fast(Features from Accelerated Segment Test), Brift(Binary Robust Independent Elementary Features) et al[4]. Where Sift and Surf method are feature detection algorithm of scale space, with the scale invariance. These algorithms have better recognition ability according to the different scenes on the PC platform, but need to be improved in order to have better recognition ability to the mobile platform is limited.

Therefore, improved Surf algorithm has been used to implement the feature points recognition and matching of mobile platform. Experiments show that the improved surf algorithm proposed in this paper is an effective and suitable method for mobile devices with augmented reality natural feature points extraction and matching algorithm, which improve the feature point recognition precision and speed.

\section{Description of SURF Algorithm}

Flow Chart of Surf Algorithm. The SURF algorithm was proposed by Bay et al., and it is a kind of fast Sift improved algorithm [5]. It is mainly divided into 3 parts: 1) Feature point extraction. That is, based on the integral image, to replace approximately two order Gauss filter with the box filtering, and to calculate the Hessian values of the selected feature points and its surrounding points, 
if it is the maximum, it is the feature point. 2) Feature point description. Haar wavelet is calculated based on the surrounding area of feature points, and the four kind of sum is calculated to form the feature description. 3) Matching with the description vector of feature points. Its biggest characteristics are using the Harr features and integral image, replacing approximately two order Gauss filter with the box filtering, and accelerating the convolution with integral images. So it reduces the time complexity of computing, and improves the velocity of calculation. Flow chart of the Surf algorithm is shown as follows:

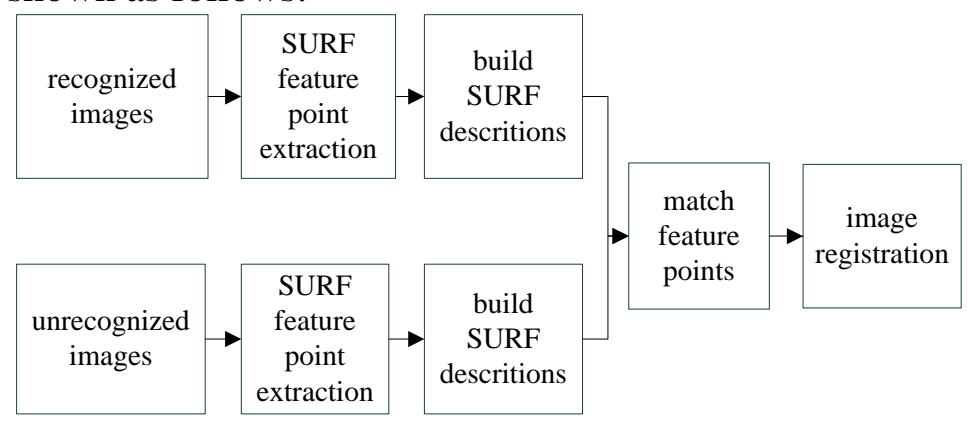

Fig.1. Flow chart of Surf algorithm

Feature Points Extraction. Integral image is a representation method for features of the original image, as shown in Figure 2 dark areas represent integral value of points $(x, y)$, and it is the sum of the gray value of dark regions. Through the integral of the original image $I(x, y)$, the integral image $I_{\Sigma}(x, y)$ is got, and $I(x, y)$ represents the pixel value, as shown in formula (1):

$$
I_{\Sigma}(x, y)=\sum_{i=0}^{i \leq x} \sum_{j=0}^{j \leq y} I(x, y)
$$

Build Fast-Hessian matrix:The point $I(x, y)$ in Fast-Hessian matrix is defined as:

$$
H(x, \sigma)=\left[\begin{array}{cc}
L_{x x}(x, \sigma) & L_{x y}(x, \sigma) \\
L_{y x}(x, \sigma) & L_{y y}(x, \sigma)
\end{array}\right]
$$

Among them, $L_{x x}$ is the convolution $g(\sigma)=\frac{1}{2 \pi \sigma^{2}} e^{-\left(x^{2}+y^{2}\right) / 2 \sigma^{2}}$ of the gray value and two order Gauss filter $\frac{\partial^{2}}{\partial X^{2}} g(\sigma)$ of $I(x, y)$, the meaning of $L_{x y}$ and $L_{y y}$ is similar. The box filtering is used to approximate Gauss differential function, and Fast-Hessian matrix is as follows:

$$
H_{\text {approx }}(x, \sigma)=\left[\begin{array}{ll}
D_{x x}(x, \sigma) & D_{x y}(x, \sigma) \\
D_{y x}(x, \sigma) & D_{y y}(x, \sigma)
\end{array}\right]
$$

Among them, the values were $D_{x x} 、 D_{x y} 、 D_{y y}$, after the block filtering template with image convolution, Happrox determinant is:

$$
\Delta H_{a p p r o x}=D_{x x} D_{y y}-\left(0.9 D_{x y}\right)
$$

Scale space building.To make the Surf algorithm invariant in scale, the SURF algorithm does the filter processing on the original image using the frame filters of different size, and it forms the image Pyramid. That is, the input image function repeats the convolution with Gauss function and repeats the two times sample on it, and this method is mainly used in the implementation of the Sift algorithm, each layer of the image depends on the previous layer image, and the image need to resize. Therefore, the amount of computation of the method is larger. Surf algorithm is different from the Sift algorithm, and it increases the size of image kernel, and the algorithm allows the scale space multi-layer images are processed at the same time, do not need two sampling on the image, so it improves the performance of the algorithm.

Feature point detection based on the neighborhood maximum.Based on the threshold of the Fast-Hessian matrix determinant the image points which meets the threshold are detected, then a 
non maximal suppression is done at 26 points around the point, then the feature point set is got, finally the $3 \mathrm{D}$ two times interpolation is done to achieve sub pixel level localization on feature points.

Feature Descriptor. In order to ensure the rotation invariance, Haar wavelet response(Haar wavelet length of $4 \sigma$ ) is firstly calculated of $\boldsymbol{x}$ and $\boldsymbol{y}$ direction which in the area that each feature point as the center, $6 \sigma$ (feature point scale value)as the radius, then make Gauss weighted on these responses that takes the feature points as the center. Using $\pi / 3$ sector as a sliding window, calculate response summation of $\boldsymbol{x}$ and $\boldsymbol{y}$ direction in sliding window, then get a local direction vector. Select the longest vector direction as the main direction of the feature points, by rotating sector window to traverse the entire circle.

First make the coordinate axis the main direction by selecting the feature point as the center, then select the square area in the main direction with the side length of $20 \sigma$ and divide this area into $4 \times 4$ sub region; calculate Haar wavelet responses of 25 normalized space sampling for each sub region. Assuming $\boldsymbol{d}_{\boldsymbol{x}}$ and $\boldsymbol{d}_{\boldsymbol{y}}$ respectively represent the Haar wavelet responses of horizontal and vertical (relative to the direction of the feature point), in order to enhance the robustness, can make Gauss filter on and take the center of filter as the feature point, sum the $d_{x}, d_{y},\left|d_{x},\right| d_{y} \mid$ in each area, then take a four dimensional feature vector $v\left(\sum d_{x}, \sum d_{y}, \Sigma\left|d_{x}\right|, \Sigma \mid d_{y}\right)$, vector of all sub regions to form the feature vector of this point, which length is 64 . The normalized feature vector of this point has rotation, scale and illumination invariant.

Feature matching.Feature point matching can be carried out when got the Surf feature vector. Search the two nearest neighbor feature point in Euclidean space by the Best Bin First(BBF) algorithm[6]. If the ratio of the nearest distance and the second near distance is less than a certain threshold, then takes the match point. The threshold is 0.65 in this paper.

\section{Improved SURF Algorithm for Mobile Devices}

According to the characteristics of the mobile terminal, the Surf was improved, so that it can adapt to the characteristics of resource constrained of mobile devices, under the premise of accuracy, improve the efficiency. Three improvements are done (1) dynamically build image Pyramid; (2) feature point filtering; (3) fast matching algorithm.

Dynamically build image Pyramid. In the augmented reality application based on mobile terminal, detected images are obtained from video stream of camera. Due to hand jitter and other reasons, there are differences in the template image size. In order to improve the feature point detection speed, the Surf algorithm associates constructed image pyramid layers with the input image's width and height, and it realizes the dynamic adaptive Pyramid layer to improve the response time. The specific method is to use the minimum of image width and height to get Pyramid layers, as shown in formula (5):

$$
O=\frac{\ln S}{C}-a
$$

Among them, $\mathrm{S}$ is the minimum of length and width of the image; $\mathrm{C}$ is constant; $\boldsymbol{a}$ is the threshold to ensure that the Pyramid layer number is within a certain scope. The value of constant $\mathrm{C}$ is 1.5 in the project, $\boldsymbol{a}$ is 2 .

Feature points filtering.The Surf algorithm has more feature points and their distribution is not uniform, and it will cause the problem of local optimum. Under the circumstances of distance constraints, it cannot be guaranteed that the optimal feature points are inside the optimal set, and it fall into the local optimal. So in this project, the improved Surf algorithm filters the feature points. Firstly, the corresponding Harr is calculated on the feature points within a certain radius, and some ones less than a certain threshold are directly filtered, then the niche genetic algorithm is introduced[7] to get rid of similar individuals.

The distance between two feature points $\bar{D} i j$ is calculated as the formulas (6)、(7): 


$$
\begin{aligned}
& \left\|\boldsymbol{x}_{i}-x_{j}\right\|=\sqrt{\sum_{k=1}^{S}\left(x_{i_{k}}-x_{j_{k}}\right)^{2}} \quad \begin{array}{c}
i=1,2, \ldots, M+N-1 \\
j=i+1, \ldots, M+N
\end{array} \\
& \bar{D} i j=\frac{\left\|x_{i}-x_{j}\right\|}{S}
\end{aligned}
$$

If $0<\bar{D}_{i j}<\varepsilon$ ( $\varepsilon$ determines the scope of similarity, specific value is set according to the situation), it shows that the two individuals are similar, in the comparison of the Harr of two feature points, a penalty function is imposed to the small feature point to reduce the characteristics content. Penalty function for the project: $f \min \left(x_{i}, x_{j}\right)=$ Penalty

Classification and optimization matching.First of all, according to the Hessian matrix trace the descriptors are classified. If $t$ is greater than 0 the descriptor will be put in a "positive" classification, if $t$ is less than 0 , it will be put in the "negative" classification. In the comparison of descriptors, first its classification is compared, if they are not in a big classification they are not further compared. The BBF algorithm with classification optimization speeds up the matching speed, and reduces the amount of computation and the occupied memory for the mobile device, and improves performance. According to the existing mismatch problem in the feature point matching, RANSAC [8] method will remove match point of poor quality as the outer points.

\section{Experimental Analysis}

Using iPhone as the test platform which has the iOS operating system, making picture using its own rear camera, using the resolution of $640 * 480$, development tools is Xcode 5.0.2, taking simulation experiment for surf and improved Surf. The image to be recognized is shown in Fig 2.

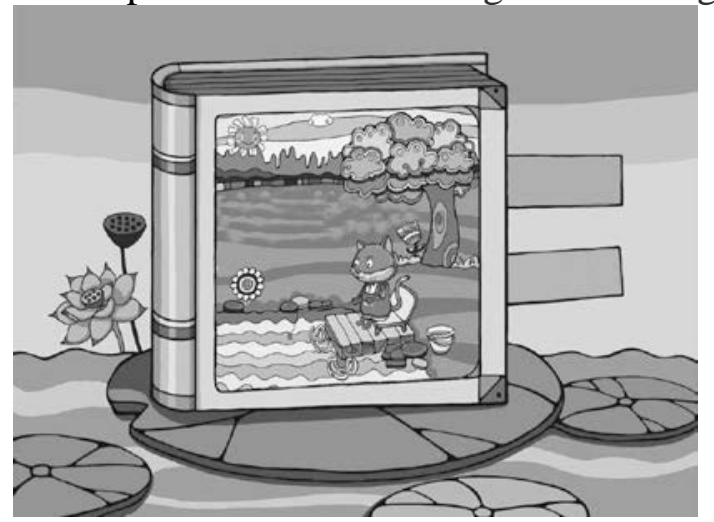

Fig.2 The Image to be Recognized

The compared simulation experiment is made for original picture, rotate 30 degrees picture, adding Gauss noise picture, the consumption of time and the number of matched feature point is shown in table 1.

Table 1. Feature points matching.

\begin{tabular}{cllllll}
\hline Detection & \multicolumn{2}{c}{ Original } & \multicolumn{2}{l}{ rotate 30 degrees } & \multicolumn{2}{c}{ Adding Gauss noise } \\
\cline { 2 - 7 } algorithm & Surf & improved Surf & Surf & Improved Surf & Surf & improved Surf \\
\hline $\begin{array}{c}\text { Matched } \\
\text { feature }\end{array}$ & 93 & 68 & 113 & 86 & 154 & 109 \\
points & & & & & \\
\hline $\begin{array}{c}\text { Detection } \\
\text { time(ms) }\end{array}$ & 667 & 514 & 976 & 717 & 1409 & 983 \\
\hline
\end{tabular}

\section{Conclusion}

The Surf algorithm is more commonly feature point recognition and matching algorithm. Firstly, the basic principle and characteristics of Surf is introduced in this paper; then Surf algorithm is improved to feature extraction and matching according to the characteristics of resource constrained 
in mobile devices. The dynamic Pyramid is used in order to real-time requirement of augmented reality based on mobile device. Then niche genetic idea is introduced to increase the distinctive feature points and reduce the similar feature points. Last BBF algorithm is optimized to speed up the feature matching speed. The experiment based on iPhone mobile(iOS platform) shows that the improved Surf proposed in this paper is an effective mobile equipment image detection and recognition algorithm which the speed and accuracy of image detection, and lays the foundation for video, animation, text, 3D virtual scene registration of mobile augmented reality technology.

\section{Acknowledgement}

In this paper, the research was sponsored by the General Program of Science and Technology Development Project of Beijing Municipal Commission of Education (Project No. KM201410012001) and National Science and Technology Supporting Funding Project(Project No. 2013BAH41F02) and Youth Innovation Fund Project of science of Beijing Institute Of Fashion Technology (Project No.2014AL-29) and Beijing Talent Strong School Plan -- the Innovation Team Project(Project No. TTBIFT_TD_002) and Beijing Key Laboratory of Digital and Interactive Media Project (Project No. KF2013-13).

\section{References}

[1] R Azuma, Y Baillot, R Behringer, etc. Recent Advances in Augmented Reality [J]. Computer Graphics and Applications(S0272-1716), 2001, 21(6): 34-47.

[2] Yawei Luo, Benchang Wei, Tao Guan etc. Research on a fast feature match method for mobile devices[J]. Application Research of computers, 2013, 30(02): 591-594.

[3] Fen Huang, Yu Zhou, Yao Yu,etc. Piano AR: a markerless augmented reality based piano teaching system [C] / /Proc of Intelligent Human-Machine Systems and Cybernetics. 2011: 47-52.

[4] CALONDER. M, LEPETIT. V, STRECHA. C. Brief: Binaryrobust independent elementary features[J]. Computer Vision, September 2010: 778-792

[5] Valgren Christoffer, Lilienthal Achim. SIFT, SURF and Seasons: Long-Term Outdoor Localization Using Local Features[EB/OL].[2010-08-10]. http://www.aass.oru.se/Research/Learning/publications/Valgren_and_Lilienthal_2007-ECMR07-SI FT_SURF_and_Seasons.html.

[6] Xiaodan Chen, Yuren Du, Xiubin Gao. A fast algorithm of image feature points matching based on SURF[J].Journal of Yangzhou University(Natural Science Edition),2012, 15(4), 64-67.

[7] Zhiyi Zhang, Rongxiang Yuan etc. Application of Self-adaptive Niching Genetic Algorithm in System Level Synthesis. Computer Engineering[J]. Computer Engineering, 2008(08), 221 222.

[8] Min Yang, Chunlin Shen. A random sampling algorithm for fundamental matrix robust estimation [J]. Journal of Applied Sciences, 2004, 22(2): 178-182. 